\title{
Biological Studies of Angiotensin II Analogs Using Nanostructured Micelles
}

\author{
Cibele Nicolaski Pedron, Daniele Ribeiro Araújo, and Vani Xavier Oliveira Jr. \\ Centro de Ciências Naturais e Humanas, Universidade Federal do ABC, Santo André, 09210580, Brazil
}

\section{Introduction}

Malaria is an acute infectious disease caused by the protozoa of the genus Plasmodium. In humans, five species are responsible for malaria: P. falciparum, P. vivax, P. ovale, P. malariae and P. knowlesi. Due to resistance to antimalarial drugs, it is important to develop new treatments including peptides. The antiplasmodial action of angiotensin II $\left(\mathrm{H}_{3} \mathrm{~N}^{+}\right.$-Asp-Arg-Val-Tyr-Ile-His-Pro-Phe-COO $\left.{ }^{-}\right)$and its analogs has been reported. It inhibits the development of Plasmodium gallinaceum in Aedes aegypti mosquitoes $[1,2]$.

To protect the peptides from protease action and promote sustained release, thermoreversible synthetic copolymers can be used as a drug delivery system. A drug delivery system is defined as a formulation or device that allows the administration of a therapeutic substance and provides better efficacy and safety by controlling the speed, time and place of release of the drug in the body [3]. This study describes the encapsulation of the antiplasmodial analogs of angiotensin II via preparation of formulations for slow release of peptides aiming for intramuscular administration.

\section{Results and Discussion}

The peptides were manually synthesized in the solid-phase method using Fmoc strategy and Wang and Rink Amide resins. The couplings are successful and used the coupling agents $N, N^{\prime}$-diisopropylcarbodiimide (DIC) and $N$-hydroxybenzotriazole (HOBt). The syntheses were satisfactory with the peptides resulting a chromatographic purity higher than 95\%; mass characterization by LC/MS-ESI confirmed that the purified peptides agreed well the expected theoretical values (Table 1).

Thermoreversible hydrogels from Poloxamer (PL) are efficient systems for the prolonged release of drugs. These were prepared at concentrations of PL 407 25\%, PL $40723 \%$ + PL $4032 \%$ and PL $40723 \%$ + PL 188 2\%. Mixed micellar systems are formed by mixing two binary copolymers and exhibit synergistic properties including increased stability of the micelles and efficient drug encapsulation versus systems with only one copolymer $[4,5]$.

The peptide-poloxamer characterization was performed by photon correlation spectroscopy. At $25^{\circ} \mathrm{C}$, the formulation showed an average diameter ranging from 33 to $225 \mathrm{~nm}$. At $37^{\circ} \mathrm{C}$, the diameter decreased to 21- $40 \mathrm{~nm}$ (Table 2) proving the aggregation and micelle formation at body temperature. With the incorporation of peptides, the average micelle diameter was not changed at either temperature. The polydispersity index ranged from 0.1 to 0.4 indicating a homogeneous particle population.

The in vitro permeation assays were carried out with a vertical diffusion Franz-type cell. The receptor medium was Hepes buffer, and the membranes were cellulose acetate. The peptide $\mathrm{H}_{3} \mathrm{~N}^{+}-\mathrm{Val}-$ Tyr-Ile-His-Pro-Phe-COO- had a release profile that was similar to the three formulations. The $24 \mathrm{~h}$ release concentrations of PL 407, PL $407+$ PL 403 and PL $407+$ PL 188 were $61.5 \pm 1.8 \mu \mathrm{g} / \mathrm{mL}, 67.0$ $\pm 10.6 \mu \mathrm{g} / \mathrm{mL}$ and $50.3 \pm 4.8 \mu \mathrm{g} / \mathrm{mL}$, respectively.

The release results showed that release flow for poloxamer formulations were lower than that obtained for free peptide. The sequence VYIHPF in the poloxamer systems formed by PL 407, PL 407 + PL 403 and PL 407 + PL188 released 51, 56 and 42\%, respectively, relative to free peptide. This indicates that these systems are effective models for this class of compound. 
Table 1. Analogs characterization.

\begin{tabular}{|c|c|c|c|c|}
\hline & Peptide & $\begin{array}{l}\text { Purity } \\
\text { HPLC }^{a}\end{array}$ & $\begin{array}{c}\text { Molecular weight } \\
(M W)\end{array}$ & $\left(m+H^{+}\right)^{b}$ \\
\hline 1 & $\mathrm{H}_{3} \mathrm{~N}^{+}$-Asp-Arg-Val-Tyr-Ile-His-Pro-Phe-CONH & $99 \%$ & 1045.5 & 1047 \\
\hline 2 & $\mathrm{H}_{3} \mathrm{~N}^{+}$-Asp-Arg-Val-Tyr-His-Ile-Pro-Phe- $\mathrm{CONH}_{2}$ & $99 \%$ & 1045.5 & 1047 \\
\hline 3 & $\mathrm{H}_{3} \mathrm{~N}^{+}$-Tyr-His-Pro-Phe- $\mathrm{CONH}_{2}$ & $99 \%$ & 562.2 & 563 \\
\hline 4 & $\mathrm{H}_{3} \mathrm{~N}^{+}$-Ile-His-Pro-Phe- $\mathrm{CONH}_{2}$ & $98 \%$ & 512.3 & 513 \\
\hline 5 & $\mathrm{H}_{3} \mathrm{~N}^{+}$-Val-Tyr-Ile-His-Pro- $\mathrm{CONH}_{2}$ & $99 \%$ & 627.3 & 629 \\
\hline 6 & $\mathrm{H}_{3} \mathrm{~N}^{+}$-Val-Tyr-Ile-His-Pro-Phe-COO- & $98 \%$ & 774.4 & 775 \\
\hline 7 & $\mathrm{H}_{3} \mathrm{~N}^{+}$-Asp-Arg-Val-Tyr-Ile-His-Pro-Phe-COO- & $99 \%$ & 979.5 & 980 \\
\hline
\end{tabular}

${ }^{a} H P L C$ profiles were obtained under the following conditions: Column Supelcosil $C_{18}(4.6 \times 150$ mm), 60A, $5 \mu \mathrm{m}$; Solvent System: A: 0.1\% TFA/ $\mathrm{H}_{2} \mathrm{O}, \mathrm{B}: 0.1 \% \mathrm{TFA}$ in $60 \% \mathrm{ACN} / \mathrm{H}_{2} \mathrm{O}$; Gradient: 5 $95 \% \mathrm{~B}$ in 30 minutes; Flow: $1.0 \mathrm{~mL} / \mathrm{min} ; \lambda=220 \mathrm{~nm}$; Injection volume: $50 \mu \mathrm{L}$ and sample concentration: $1.0 \mathrm{mg} . \mathrm{mL}^{-1}$; ${ }^{b}$ Mass measurements were performed in a positive mode with the following parameters: Column Waters Nova-Pak $C_{18}(2.1 \times 150 \mathrm{~mm}), 60 \AA$ A $3.5 \mu \mathrm{m}$; Solvent System: A: $0.1 \%$ TFA $/ \mathrm{H}_{2} \mathrm{O}, \mathrm{B}: 0.1 \% \mathrm{TFA}$ in $60 \% \mathrm{ACN} / \mathrm{H}_{2} \mathrm{O}$; Gradient: $5-95 \% \mathrm{~B}$ in 30 minutes; Flow:0.4 $\mathrm{mL} / \mathrm{min} ; \lambda=220 \mathrm{~nm}$; Injection volume: $30 \mu \mathrm{L}$; Sample concentration: $1.0 \mathrm{mg} . \mathrm{mL}^{-1}$; mass range between 200-2000 Daltons.

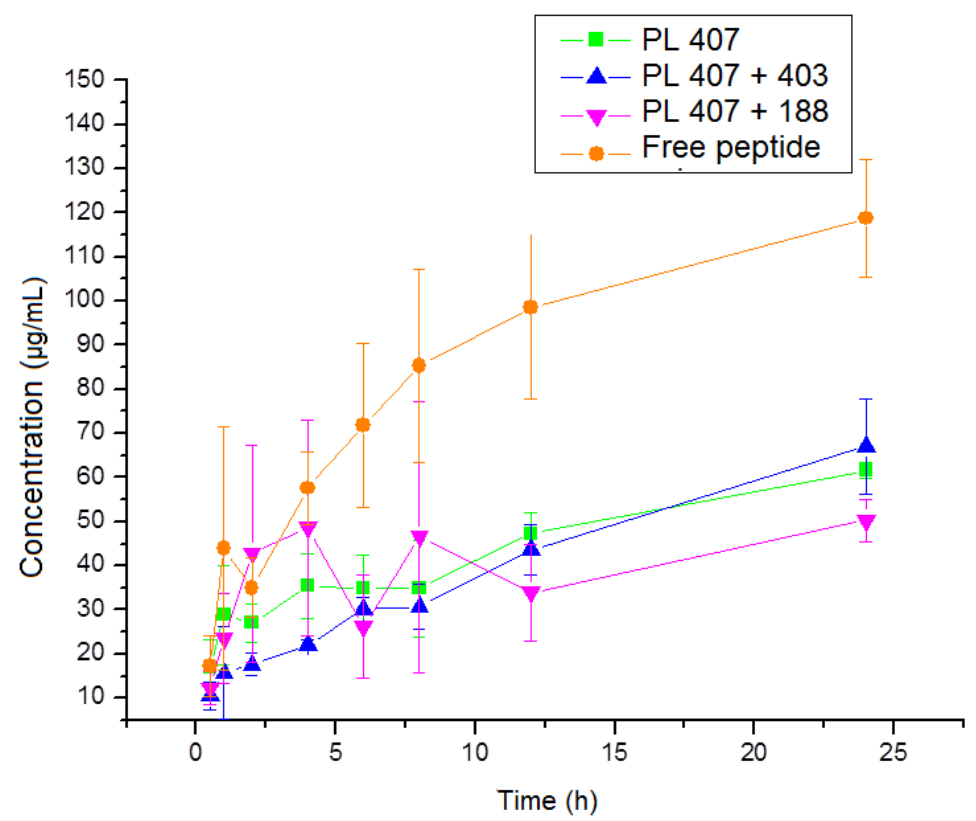

Fig. 1. Release profile of the formulations and peptide $\mathrm{H}_{3} \mathrm{~N}^{+}$-Val-Tyr-Ile-His-Pro-Phe-COO- 
Table 2. Diameter and size distribution percentage obtained at 25 and $37^{\circ} \mathrm{C}$.

\begin{tabular}{ccccc}
\hline & \multicolumn{2}{c}{$25^{\circ} \mathrm{C}$} & $37^{\circ} \mathrm{C}$ \\
\cline { 2 - 5 } & Diameter & \% Intensity & Diameter & \% Intensity \\
\hline PL 407 & $43 \pm 0.9$ & $86 \pm 0.7$ & $21 \pm 0.1$ & $100 \pm 0.0$ \\
PL 407 + PL 403 & $39 \pm 0.4$ & $96 \pm 3.5$ & $37 \pm 1.1$ & $97 \pm 0.9$ \\
PL 407 + Peptide 2 & $45 \pm 0.5$ & $84 \pm 0.3$ & $40 \pm 0.7$ & $97 \pm 2.5$ \\
PL 407 + PL 403 + Peptide 2 & $39 \pm 0.8$ & $93 \pm 0.7$ & $36 \pm 0.5$ & $74 \pm 0.6$ \\
PL 407 + PL 188 + Peptide 2 & $41 \pm 1.3$ & $84 \pm 0.6$ & $25 \pm 2.0$ & $100 \pm 0.2$ \\
PL 407 + Peptide 3 & $52 \pm 4.1$ & $81 \pm 4.1$ & $24 \pm 2.1$ & $100 \pm 0.4$ \\
PL 407 + PL 403 + Peptide 3 & $54 \pm 2.2$ & $91 \pm 0.2$ & $35 \pm 2.0$ & $98 \pm 0.5$ \\
PL 407 + PL 188 + Peptide 3 & $62 \pm 3.9$ & $76 \pm 2.5$ & $25 \pm 1.5$ & $100 \pm 0.0$ \\
PL 407 + Peptide 6 & $188 \pm 20$ & $68 \pm 2.2$ & $27 \pm 0.5$ & $99 \pm 0.7$ \\
PL 407 + PL 403 + Peptide 6 & $33 \pm 1.0$ & $68 \pm 1.2$ & $27 \pm 1.4$ & $99 \pm 1.1$ \\
PL 407 + PL 188 + Peptide 6 & $197 \pm 31$ & $74 \pm 5.0$ & $28 \pm 0.9$ & $97 \pm 0.6$ \\
PL 407 + Peptide 7 & $170 \pm 14$ & $77 \pm 2.9$ & $28 \pm 0.5$ & $94 \pm 3.2$ \\
PL 407 + PL 403 + Peptide 7 & $34 \pm 1.5$ & $68 \pm 2.5$ & $32 \pm 0.7$ & $86 \pm 0.8$ \\
PL 407 + PL 188 + Peptide 7 & $225 \pm 69$ & $74 \pm 25$ & $35 \pm 1.8$ & $82 \pm 1.2$ \\
\hline
\end{tabular}

Data presented as (mean $\pm S D, n=3$ ). The measurements of the hydrodynamic diameter was performed by Zetasizer particle analyzer ZS (Malvern ${ }^{\circledR}$ Instruments) at an angle of $173^{\circ}$ at temperatures of 25 and $37^{\circ} \mathrm{C}$.

\section{Acknowledgments}

We thank Universidade Federal do ABC (UFABC), Universidade de São Paulo (USP), Universidade Federal de São Paulo (UNIFESP), Coordenação de Aperfeiçoamento de Pessoal de Nível Superior (CAPES) and Fundação de Amparo à Pesquisa do Estado de São Paulo (FAPESP).

\section{References}

1. Maciel, C., et al. PLoS ONE 3, e3296 (2008), http://dx.doi.org/10.1371/journal.pone.0003296

2. Silva, A.F., et al. J. Pept. Science 20, 640-648 (2014), http://dx.doi.org/10.1002/psc.2641

3. Jain, K.K., Methods Mol. Biol. 437, 1-50 (2008), http://dx.doi.org/10.1007/978-1-59745-210-6_1

4. Kulthe, S.S., et al. Colloids Surf. B:Biointerfaces 88, 691-696 (2011), http://dx.doi.org/10.1016/i.colsurfb.2011.08.002

5. Lee, E.S., et al. Colloids Surf. B:Biointerfaces 82, 190-195 (2011), http://dx.doi.org/10.1016/j.colsurfb.2010.08.033 